\title{
Marketing como herramienta para la gestión. El caso de la ruta del vino de la D.O. Empordà (Costa Brava, España)
}

\section{Marketing as a tool for territorial management. The case of Emporda wine route (Costa Brava, Spain)}

\author{
Autoras: \\ Marta Casas i Serrabassa \\ Neus Crous-Costa
}

\section{Resumen}

El objetivo de este texto es presentar el marketing como una herramienta de gestión territorial turística. Así, el artículo se estructura en tres apartados bien diferenciados. Primero, la revisión teórica parte de las teorías más ampliamente conocidas y aceptadas de la mercadotecnia se llega al concepto actual de marketing holístico. A continuación, la segunda parte propone los ámbitos en los que puede aplicarse realmente el marketing como gestión. Tercero, se muestra el caso de estudio de la Ruta del Vino D.O. Empordà. La investigación se estructura como un caso de estudio que combina la revisión bibliográfica con un enfoque puramente empirista. Finalmente, en las recapitulaciones finales se hace hincapié en algunos de los aspectos de la gestión marketiniana que el caso de estudio confirma más claramente. El presente texto no está exento de limitaciones, entre las que podemos destacar la escasa bibliografía por lo que respecta a marketing como herramienta de gestión, gap que pretendemos ayudar a estrechar, si bien debe considerarse que se trata de un caso de estudio regional al que deberán sumarse otros para poder desarrollar un verdadero ensayo teórico.

\section{Abstract}

The objective of this text is to present marketing as a tool for tourist territorial management. Thus, the article is divided into three well differentiated sections. First, the theoretical review starts from the most widely known and accepted theories of marketing and comes to the current concept of holistic marketing. Next, the second part proposes the areas in which marketing can really be applied as management. The third part focuses on the case study of the Wine Route D.O. Empordà. The research is structured as a case study that combines a bibliographic review with a purely empiricist approach. Finally, the final recaps emphasize some of the aspects of marketing management that the case study confirms most clearly. This text is not without limitations, among which we can highlight the scarce bibliography regarding marketing as a management tool, a gap that we intend to help narrow, although it should be considered that it is a regional case study that others must be added in order to achieve a true theoretical essay.

Keywords: Costa Brava, marketing club, marketing as a management tool, tourism, wine route

Palabras clave: club de producto, Costa Brava, marketing como herramienta de gestión, ruta del vino, turismo 


\section{Introduction}

Resulta prácticamente innecesario decir que el turismo es una actividad eminentemente territorial, ya que se desarrolla en un destino completo que puede abarcar un área geográfica más o menos amplia.

Actualmente existe una cantidad creciente de casos de estudio referidos al desempeño de esta actividad en el ámbito urbano y algunos trabajos también en el ámbito rural. Sin embargo, la atención que el mundo académico ha dedicado a las rutas es menor. Algunos ejemplos de ello son escritos sobre desarrollo y gestión (Consejo de Europa, 2015), gobernanza (Llorca Hernández, 2018), la recuperación y diseminación de patrimonios frágiles como la memoria histórica (González Vázquez, 2018), geopolítica (Amilhat-Szary y Guyol, 2009), y marketing (European Travel Commission y Organización Mundial del Turismo, 2017), entre otros temas. Aun así, las rutas estructuradas desde las distintas organizaciones de marketing y/o gestión de destino (DMO, por sus siglas en inglés) resultan interesantes como esfuerzo vertebrador de un territorio. Partiendo de un recurso normalmente patrimonial (cultural, natural o inmaterial), pretende crear o diseminar flujos de visitantes de tal manera que esponjando las externalidades negativas sus efectos acaben resultando menos dañinos. A la vez, se aumenta el número de personas que se benefician de los impactos positivos que no pueden reducirse a los ingresos económicos.

Esta investigación pretende abordar la cuestión de las rutas desde el punto de vista del marketing holístico utilizado como herramienta para la gestión integral de un territorio, hasta donde la posibilitan las facultades propias de una DMO como es el Patronato de Turismo Costa Brava Girona.

Conviene puntualizar que para los fines de este texto utilizaremos el término ruta para referinos a los itinerarios de cualquier temática que se desarrollan fuera del ámbito urbano, es decir, en la naturaleza, el entorno rural o poblaciones pequeñas y medianas.

\section{Objetivos y metodología}

El objetivo de este texto es presentar el marketing como una herramienta de gestión territorial turística. Partiendo de las teorías más ampliamente conocidas y aceptadas se presenta una recapitulación cuya conclusión está recogida en buena medida en el concepto actual de marketing holístico: marketing no puede reducirse a un mero sinónimo de generación de propaganda. En base a este enfoque, se proponen algunos ámbitos de actuación real para que la mercadotecnia pueda ser también un sistema de gestión. A través del caso de estudio de la Ruta del Vino Denominación de Origen (D.O.) Empordà se pretende hacer una identificación de fórmulas en las que el conjunto de herramientas relacionadas con la mercadotecnia pueden servir para la gestión de un producto turístico, entendiendo éste como una capa más del territorio humanizado.

Dicho caso de estudio se ha construido desde un punto de vista eminentemente empirista y en primera persona. Así, se describen las acciones llevadas a cabo por el ente de promoción de la província de Girona, el Patronato de Turismo Girona Costa Brava, concretamente a través de su producto Ruta del Vino del Empordà.

\section{El concepto de marketing. Una recapitulación}

En el lenguaje corriente se ha hecho común, incluso en el profesional, utilizar el término marketing como un sinónimo de promoción y comunicación. Este reduccionismo no solamente es un error conceptual, sino que en la práctica limita el campo de visión y, por tanto, el abordaje creativo de las distintas situa- 
ciones. Así, las llamadas acciones de marketing no están solamente enfocadas a dar a conocer un bien (producto o servicio). De acuerdo con Ellis et al. (2010) esta metonimia puede deberse al foco que ya en la primera mitad del siglo XX se puso en la generación y la activación de la demanda. Estrictamente, su vocación se amplía al diseño de producto, el conseguir ventajas competitivas y las relaciones con los públicos, por ejemplo.

En la actualidad se ha desarrollado una cantidad muy considerable de teoría respecto al marketing y, específicamente, al marketing turístico. Aun así, posiblemente la más ampliamente conocida, utilizada como fundamento, sea la formulada por Philip Kotler en la década de 1960 (Kotler y Armstrong, 2008). Ésta se desarrolló fundamentalmente desde el punto de vista de la oferta, de tal modo que el marketing mix se componía de las famosas " $4 \mathrm{p}$ ": producto, precio, distribución (placement) y promoción. Idealmente los departamentos de marketing deberían ocuparse o participar en el diseño y materialización de todos los componentes anteriores de tal modo que la marca corresponda con lo que se vende, alineándose también con las características del mercado y el comportamiento del consumidor.

En el campo del turismo un ejemplo de esto lo constituyen las estrategias de marca país o de marca ciudad: incluye la construcción de un imaginario alrededor de este ámbito geográfico, a la vez que incide, o debería incidir, en el constructo social (Carrillo Sánchez y García Calderón, 2019) y en características del producto. Dado que el producto es el propio lugar, sus componentes tienen que ver con las infraestructuras materiales (diseño urbano, red de transportes...) así como los valores inmateriales (construcción de la identidad colectiva...), que, de hecho, afectan primeramente a los residentes.En los años noventa fue paradigmático el caso de la Barcelona olímpica (Morillo Palomo, 2016, entre muchos otros). Más cercano es el caso de la marca Perú, que además del impacto internacional logró una amplia adhesión entre la población peruana (Schmitz y Espinosa, 2015), mejorando la autoestima a base de valorar los atributos de la comunidad nacional.

Focalizándonos en el marketing de servicios, Booms y Bitner (en Ivy, 2008) añadieron otras "3p" a las cuatro originales: el soporte físico (physical evidence: el espacio donde el personal y los clientes interactúan), la gente (people: todos los actores y sus interacciones) y los procedimientos a través de los cuales se presta un servicio.

Así pues, se ha pasado a subrayar el elemento humano. En relación a esto, en el contexto turístico actual, además del "turista" y el "trabajador" como entes económicos deben tenerse en cuenta también sus equivalentes como grupo humano según la relación con el territorio.

Por un lado, tenemos a los visitantes. Una parte del corpus académico muestra cómo la imagen preconcebida de un destino llega a influenciar profundamente su comportamiento una vez llega al lugar (por ejemplo: Pearce, 2005). Sin embargo, también vale la pena remarcar que muchos de estos estudios utilizan el término comportamiento (behaviour) para referirse a la propensión de seleccionar o repetir la elección de un destino (por ejemplo: Barroso Castro, Martín Armario y Martín Ruiz, 2007; Afshardoost y Eshaghi, 2020).En los últimos años la prensa ha dado buena cuenta de situaciones como las de Cracovia (Polonia) o York (Inglaterra): a pesar de ser ciudades con un importante patrimonio histórico, son percibidas internacionalmente como escenarios para celebrar despedidas de soltero. El número de personas que viaja a ciudades como estas causando, inconscientemente, problemas de convivencia y gasto público aumenta, mientras que la imagen del lugar se deteriora atrayendo a menos personas con un interés en 
el patrimonio (que, cabe esperar, generarán más impactos positivos que negativos). La imagen turística, por tanto, tiene un impacto real e inmediato en la vida urbana.

Otro enfoque, del que se habla menos, pero igualmente cierto, nos lo aporta Chias (2004). Este consultor y profesor daba al turismo el epíteto de "el negocio de la felicidad", habiendo observado que el viaje, más concretamente el sentimiento de descubrimiento por estar en un lugar nuevo, genera felicidad en quien lo realiza. Para él tanto el patrimonio natural como el cultural son un elemento fundamental en la cadena de valor de este sector. Así, alaba brevemente la tarea del Patrimonio de la Humanidad de la UNESCO, ya que debería facilitar la preservación de los recursos del planeta así como la historia de la humanidad, a la vez que reconoce en el término "Patrimonio de la Humanidad" una etiqueta capaz de generar flujos de visitantes. A día de hoy debe reconocerse que esta es una idea controvertida, con algunos lugares sufriendo de hipo- o hiper- turistificación (du Cros, 2008; Barron, 2017). Regresando a la felicidad, si ésta no surge (solamente) de un reconocimiento social asociado a la posibilidad de haber viajado lejos y extensamente, sino de una sincera capacidad de asombro frente a la otredad, es ineludible para las autoridades públicas así como para la industria turística la preservación de las fuentes que la contienen: otras personas (diversidad cultural), paisajes, formas de vida cotidiana... Más todavía, para Chias el propósito del marketing mix es posibilitar una experiencia satisfactoria de al modo que preservación y felicidad (íntimamente ligada a la satisfacción) no pueden sino ir de la mano.

Por otro lado, en consonancia con esto, tenemos a la comunidad local. Gross (2011) fue quizás uno de los primeros académicos en señalar que se estaba prestando poca atención a la forma en que los residentes ven a los turistas. Unos años después los desafortunadamente y mundialmente famosos graffitis de Barcelona dejan claro que es uno de los puntos que todos los implicados en el sistema turístico deben abordar. Volviendo al análisis de este autor, presenta el caso del programa Pueblos Mágicos (México), centrándose en Oaxaca. Existe evidencia de la población local adaptándose a las dinámicas turísticas. Dado que el turismo en estos emplazamientos es una iniciativa de tipo top-down, las identidades y las prácticas culturales parece que se han moldeado para atraer a los visitantes, especialmente internacionales. De este modo se ha polarizado la perspectiva que los residentes tienen del turismo: una forma de vender el imaginario cultural del lugar (que beneficia solamente a unos cuantos y a menudo causa dependencia del turismo como única fuente de ingresos), o una forma de re-vigorizar la identidad cultural (repartiendo los beneficios a toda la comunidad).

Regresando a las ideas de Carrillo Sánchez y García Calderón (2019), todo lo expuesto hasta ahora evidencia que el desempeño en el mercado de un producto turístico no puede considerarse solamente en relación a la publicidad, sino que existen múltiples variables a tener en cuenta. Entre ellas se encuentran la satisfacción del consumidor, el lugar, la competencia y un largo etcétera.

En una línea de pensamiento más amplia, parecida a la desarrollada en este apartado, en Sheh y Sisodia (2015) compilaban una obra que tenía por objetivo replantear las bases del marketing clásico, acercándose a la caracterización del concepto de marketing holístico. En uno de los textos finales se explicita que este nuevo enfoque requiere de un pensamiento amplio y cohesivo. Esta visión incluye las famosas $4 \mathrm{p}$ para ampliarlas con las personas (en sentido ámplio), los procesos y el desempeño. Las dos últimas claramente tienen que ver con el marketing interno, el relacional y de desempeño.

El análisis propuesto en este artículo va en una dirección parecida. El apartado siguiente recoge, todavía 
a nivel teórico, una propuesta de aspectos en los que las herramientas propias del campo marketiniano pueden contribuir a la gestión turística de una ruta, entendida como una parte más de un territorio concreto.

\section{Marketing para la gestión de una ruta}

Hasta ahora se ha basado la aproximación teórica al concepto integral de marketing empleando ejemplos básicamente de ciudades patrimoniales. Esto se debe a que existe un corpus muy rico (en volumen así como en temas abordados) y en auge referido a este tipo de enclave humano, en cambio la literatura académica referida propiamente a las rutas es más bien modesta.

Con este handicap, se ha desarrollado la siguiente lista en base al conocimiento generado tanto a través de la bibliografía como de la docencia (a través de la cual se entra en contacto con el conocimiento directo de casos reales) y participación en casos existentes. No se está proponiendo un nuevo listado de "ps" kotlerianas o cuestionando las bases filosóficas del márketing. Se trata de una reflexión sobre los elementos a tener en cuenta a la hora de gestionar desde el punto de vista turístico un espacio territorial relativamente grande, que afecta a un volumen relativamente grande de personas y entornos naturales, como es una ruta.

Dado que en los últimos años el número de visitantes, excursionistas o turistas, no ha hecho sino ir en aumento (aumentando sus impactos negativos y positivos), parece ineludible que las organizaciones de marketing turístico adopten también una visión de responsabilidad por lo que respecta al desempeño integral de sus áreas de influencia.

En cualquier caso, debe entenderse como un esfuerzo exploratorio en este sentido, de tal manera que deberá ser reforzado o modificado por futuros casos de estudio o disertaciones teóricas.

En primer lugar existe un grupo de ámbitos en los que el marketing como herramienta de gestión puede incidir relacionados con el territorio, tanto a nivel humano, como físico y ecológico. Especialmente en las últimas décadas, la puesta en funcionamiento de rutas turísticas tiene que ver con cuestiones de equilibrio y regeneración territorial. Una ruta permite enlazar distintos recursos del territorio aportando un valor añadido (Elías, 2006). Tal y como ocurrió con el turismo rural, se ponen en funcionamiento rutas turísticas con la esperanza de que la presencia de visitantes ajenos al territorio traiga consigo no solamente ingresos, sino la posibilidad de desarrollar pequeños o medianos negocios que permitan desarrollar proyectos de vida de calidad en el entorno rural o poblaciones medias. En este sentido, algunos estudios ponen de manifiesto el rol que los itinerarios turísticos pueden desarrollar en la mejora de la gestión sostenible de los destinos (Aulet, 2010; Majdoub, 2010; Calderón Puerto, Arcila Garrido, López Sánchez, 2018)

Además, por lo general la recepción de visitantes pone en funcionamiento un mecanismo de psicología colectiva anterior a la turismofobia y cercano a la hospitalidad. Que nuestro patrimonio atraiga el interés de gente de fuera de nuestro entorno habitual aumenta la autoestima local, por lo menos inicialmente (Toselli, 2006; Chen, Chen, 2010). Esto por tanto se convierte en un aliciente para la conservación de paisajes, prácticas..., más allá de los meros ingresos económicos que se puedan generar al entorno de cada elemento. Esta (re)apreciación de los locales por su propia herencia contribuye también a afianzar otros valores intangibles como la identidad o asegurar la autenticidad del patrimonio (Sanchez, 2015).

Esta combinación de factores, la viabilidad económica y la valoración, propician realidades como la con- 
tinuidad en los usos tradicionales de la tierra (aquí básicamente nos referimos a los usos agrícolas), lo que influye también en cuestiones ambientales. Nogué (1992) se refiere a los agricultores como a los "jardineros del paisaje", poniendo en valor la importante tarea que realizan en la conservación del paisaje que es, por otro lado, uno de los principales atractivos para el turismo. Uno de los elementos que más se ha puesto en valor en el desarrollo del turismo gastronómico es la conexión que se establece entre el sector turístico y el sector agroalimentario, ayudando a la dinamización de este último (Timothy, 2015; Aulet, Mundet, Vidal, 2017; Aulet, Vidal, Majó, 2019).

De hecho, otra de las propiedades de las rutas turísticas que más interés suscita es su capacidad para distribuir los visitantes entre los diferentes nodos que la componen. Es decir, dado que el visitante es consciente de la existencia de más puntos de interés aquellos elementos reciben o bien menos visitas o bien durante menos tiempo, lo cual reduce la presión a la que el nodo se vería sometido. Al mismo tiempo, los nodos que inicialmente contaban con poca o nula capacidad de atracción empiezan a recibir más visitas sin que, a priori, esto deba suponer un problema (si es que se han habilitado correctamente para ser abiertos al público) (Hernandez, 2000). Esta es una forma en la que las rutas ayudan a distribuir los beneficios de recibir visitantes, a la vez que procuran reducir los efectos negativos de la presencia de humanos. De igual forma sucede con la afluencia a los negocios cercanos a los nodos, adoptando una perspectiva económica.

Finalmente, debemos mencionar también la relación entre visitantes y residentes. Por el momento se desconoce que existan rutas turísticas en las que se hayan dado verdaderos problemas de convivencia, más allá de situaciones anecdóticas. Si bien no se conocen estudios específicos al respecto, quizás un efecto indeseado asociado podría ser el aumento de la criminalidad que podría darse en lugares que reciben un altísimo número de visitantes, como algunos tramos del Camino de Santiago en el norte de la Península Ibérica.

El segundo grupo de contextos en los que el marketing es susceptible de ser empleado para la gestión territorial atañe a los propios visitantes. De hecho, la cuestión principal ya se ha mencionado antes: la articulación y promoción de un lugar como ruta (sea contínua o discontinua) pone en conocimiento del foráneo más lugares. De entrada, esto da al turista la posibilidad de tener una visión y un conocimiento más ricos del sitio en el que se encuentra, así como de lograr un mejor acomodamiento entre sus intereses y la oferta disponible. Claramente esto está sujeto a cuestiones prácticas como la accesibilidad a los lugares (transporte público, estado de los caminos...), los horarios, la existencia de entradas conjuntas, las posibles restricciones de entrada en pro de no superar la capacidad de carga del lugar, etc.(Sparks, Malady, 2006).

Asimismo, la integración en el imaginario turístico de un número mayor de lugares o la creación de un genuino interés en la persona para conocer más en profundidad el lugar puede redundar en la repetición de la visita que, veíamos, era uno de los objetivos iniciales de la mercadotecnia.

Para terminar, existen también nichos de actuación que corresponden propiamente a las administraciones públicas. Si nos referimos a los componentes clásicos del marketing mix, estaríamos hablando de la planificación y puesta en funcionamiento de características del producto como pueden ser las infraestructuras de comunicaciones, los servicios de salud y seguridad... Una vez puestos en funcionamiento éstos no benefician solamente a los visitantes y a la imagen del destino sino que, primordialmente, revierten 
en la calidad de vida de los residentes. Si bien este aspecto es el que más escapa de las manos de las DMOs, éstas pueden ejercer como lobbys de presión hacia las administraciones competentes.

\section{Caso de estudio: Ruta del Vino del Empordà}

El caso de estudio analizado se localiza en la província de Girona, en Catalunya. El Patronato de Turismo Costa Brava Girona es una entidad de promoción turística fundada en 1976 con el objetivo de ser el punto de encuentro de las administraciones con el sector turístico de la província de Girona. Su actividad se centra en tres ámbitos:

- Marketing interno

- Marketing externo

- Competitividad

En los más de 30 años de historia el Patronato ha promocionado sus dos marcas insignia, la Costa Brava y el Pirineo de Girona, en los principales mercados europeos. Actualmente, la marca Costa Brava goza de un buen posicionamiento tanto en el mercado estatal como en el internacional, gracias a su larga trayectoria turística y a su prestigio asociado. Por ejemplo, en 2018 recibió 7,5 M de turistas y un total de $25 \mathrm{M}$ de pernoctaciones en establecimientos de alojamiento reglado (Idescat, 2018).

Así, con la intención de gestionar, estructurar y consolidar mejor la oferta turística de la provincia de Girona, se crearon diferentes clubs de marketing. Estos clubs son agrupaciones voluntarias de empresarios y entidades públicas, interesados en gestionar, promocionar y comercializar un mismo producto turístico, por lo que tienen intereses, necesidades y preocupaciones comunes. La filosofía principal de estos clubs se basa en la cooperación y colaboración entre asociados públicos y privados bajo una marca común, con el objetivo, por un lado, de incrementar la calidad y competitividad del producto y, por el otro, de mejorar su comercialización.

La diversidad de la demanda exige que la oferta se adapte a las necesidades de ésta y, con este objetivo, el Patronato creó los clubs de producto, con la finalidad de segmentar la oferta y poder estructurar un plan de acciones adaptado a las características del público objetivo.

Actualmente el Patronato gestiona nueve clubs de marketing:

- Cultura e identidad

- Naturaleza y turismo activo

- Costa Brava Convention Bureau

- Ruta del Vino DO Empordà

- Enogastronomia

- Golf

- Unique - Premium

- Salud y bienestar

- Turismo deportivo

El Club de la Ruta del Vino D.O. Empordà se fundó el año 2014 con la intención de estructurar, gestionar y promocionar la oferta enoturística de la Costa Brava y, más concretamente, la vinculada con la Denominación de Origen Empordà. Los aspectos claves en el nacimiento del fenómeno enoturístico son la transformación de los hábitos de consumo del vino (aumentando el interés por conocer la cultura del 
vino), la crisis del precio del vino en muchas regiones vinícolas obligando a las bodegas a reestructurar sus modelos de negocio y la revolución del turismo con la aparición de nuevas tipologías (ecoturismo, turismo activo o rural) (Damià Serrano, 2015).

A esta tendencia turística se le sumó la apuesta por la profesionalización y la calidad de los vinos del Empordà, que despertó el interés del público por visitar las bodegas donde estos se elaboran, desarrollándose así un enoturismo incipiente. Desde el Patronato se decidió apoyar este movimiento y asumir el reto, apostando por un turismo desestacionalizador, sostenible y que fomenta el desarrollo rural. La sostenibilidad en el enoturismo se basa en 3 pilares: sostenibilidad económica, medioambiental y social (Mitchell y Hall, 2006).

Hoy en día, la Ruta del Vino D.O. Empordà está compuesta por 101 de empresas y entidades públicas, escogidas a través de criterios de calidad y profesionalidad y sometidas a auditorías periódicas, que, unidas, articulan la oferta enoturística del Empordà. Estas empresas y entidades forman, sobre el mapa de la Costa Brava, una red de nodos que, unidos entre sí por diferentes carreteras del territorio, permiten al viajero organizarse una ruta a medida en función de sus necesidades y sus intereses. El turismo gastronómico incluye un conjunto amplio de agentes implicados que cumplen distintas funciones como las de producción, tratamiento, almacenaje, transporte, cocina, preparación, etc. (Leal Londoño, 2013). Entre ellos hay que contemplar productores y representantes del sector agroalimentario, representantes del sector turístico como alojamiento, transporte y restauración, junto con entidades de promoción (OMT, 2019).

El concepto de Ruta del Vino como producto turístico y, al mismo tiempo, como manera de entender o ordenar un territorio, viene avalado desde hace años por iniciativas como la de Rutas del Vino de España, nacido en 2001 y actualmente con 30 regiones enoturísticas plenamente consolidadas como La Rioja, Ribera del Duero o Rueda; o las Strade del Vino en Italia, que aglutinan desde 1999 a 22 rutas enoturísticas. En este contexto, el enoturismo es entendido como una actividad turística mucho más compleja que visitar bodegas y catar vinos, ya que la principal anomalía del enoturismo en España es la obsesión con la visita a la bodega (Tolosa, 2017).

Así pues, las Rutas de Vino se organizan como un cupaje donde aparecen muchas tipologías diferentes de empresas y entidades: desde las bodegas a restaurantes, alojamientos de diferente naturaleza, tiendas y bares de vinos, empresas de actividades, sommeliers personales, museos, oficinas de turismo, etc. Todo este sector necesita personal formado y de calidad, ayuda a fijar la población en áreas rurales antes con problemas de despoblación y protege el uso agrario de la tierra.

Además, la actividad enoturística en sí ha evolucionado de manera notable, creando experiencias y propuestas memorables que cada vez atraen a más público, no necesariamente amante de los vinos, y su influencia e impacto positivo va mucho más allá de las bodegas: el enoturismo acerca al visitante a la naturaleza, la cultura y la gastronomía de la región. 


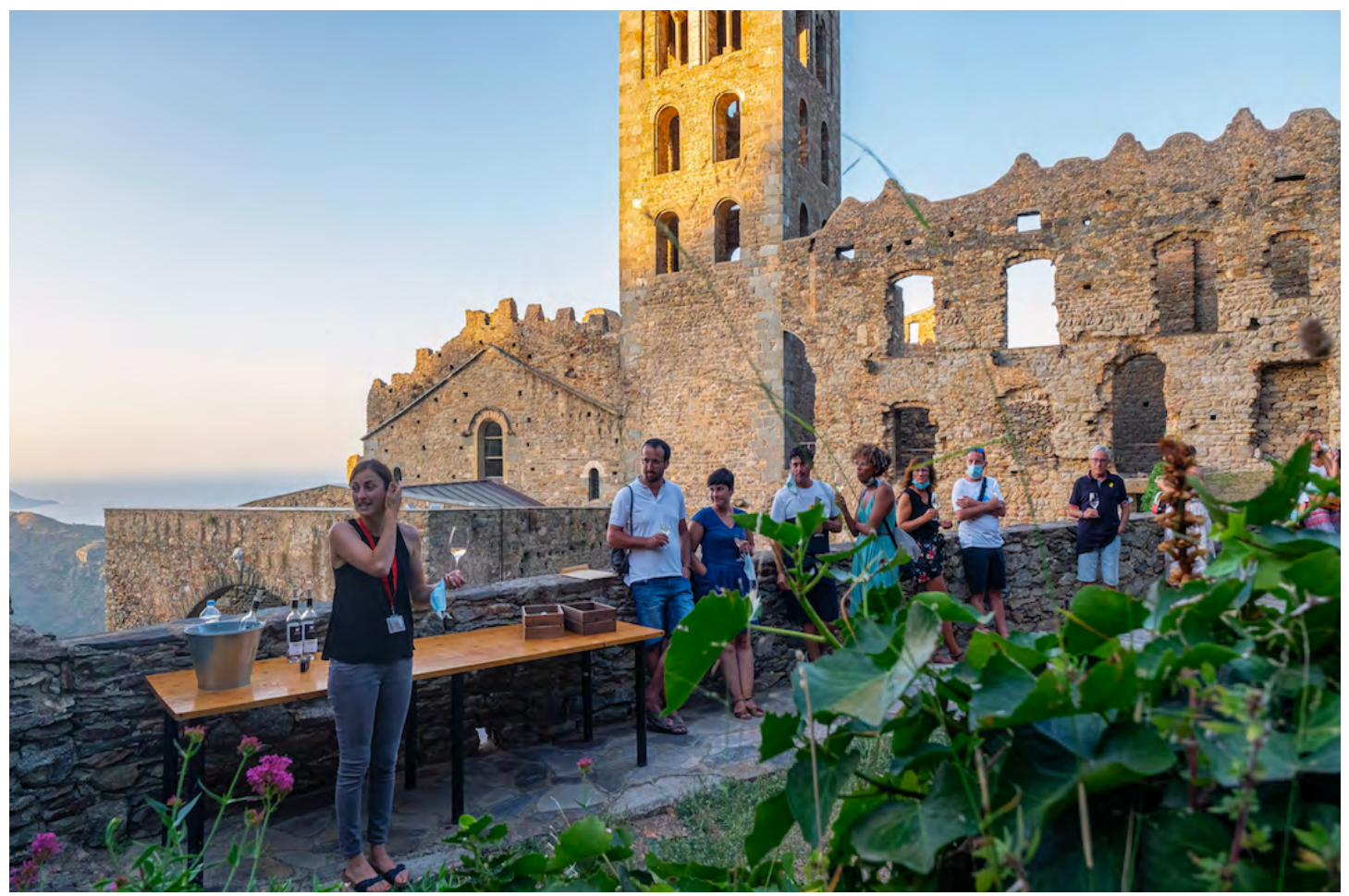

Foto 1/ Visita enoturística. Monasterio Sant Pere de Rodes. Jordi Renart. Arxiu Imatges PTCBG. 2020

\subsection{Análisis de la Ruta del Vino D.O. Empordà}

Desde su creación en el 2014 el Patronato de Turismo Costa Brava Girona recopila datos de interés sobre los visitantes de la Ruta del Vino D.O. Empordà a través de las bodegas asociadas.

En primer lugar, se puede documentar su crecimiento constante pero sostenible, tanto en volumen de bodegas adheridas como en visitantes totales, tal como se puede observar en la tabla 1.

\begin{tabular}{|l|c|c|c|}
\hline AÑO & NÚMERO DE BODEGAS & TOTAL VISITANTES ANUAL & EVOLUCIÓN \\
\hline 2014 & 14 & 35.425 & \\
\hline 2015 & 25 & 39.073 & $+10,30 \%$ \\
\hline 2016 & 26 & 47.350 & $+21,18 \%$ \\
\hline 2017 & 26 & 50.297 & $+6,22 \%$ \\
\hline 2018 & 27 & 53.450 & $+6,27 \%$ \\
\hline 2019 & 28 & 56.173 & $+5,09 \%$ \\
\hline
\end{tabular}

Tabla 1/ Evolución número de bodegas y visitantes Ruta del Vino D.O. Empordà. Elaboración propia. 2020

Actualmente hay 51 bodegas produciendo bajo el sello de la D.O y, teniendo en cuenta que el año 2020 la Ruta del Vino D.O. Empordà cuenta ya con 31 de estas bodegas asociadas, se puede afirmar que el grado 
de interés que despierta el proyecto entre los viticultores es alto: un 60,78\% de sus bodegas están adheridas a la Ruta del Vino D.O. Empordà. En general, pues, las bodegas se sienten identificadas con esta iniciativa y entienden el enoturismo como una fuente de ingresos a tener en cuenta para sus negocios.

Además, el número de visitantes se mantiene al alza, pero de manera sostenida y coherente con las capacidades de carga del territorio, donde la mayoría de bodegas y demás empresas son pequeñas. También cabe destacar el aumento significativo del gasto medio por visitante en la tienda de la bodega, debido tanto a la creación de productos enoturísticos más elaborados y por tanto de mayor precio; como a la apuesta por la elaboración de vinos de calidad, también de mayor coste (gràfico 1).

\section{Evolución gasto medio}

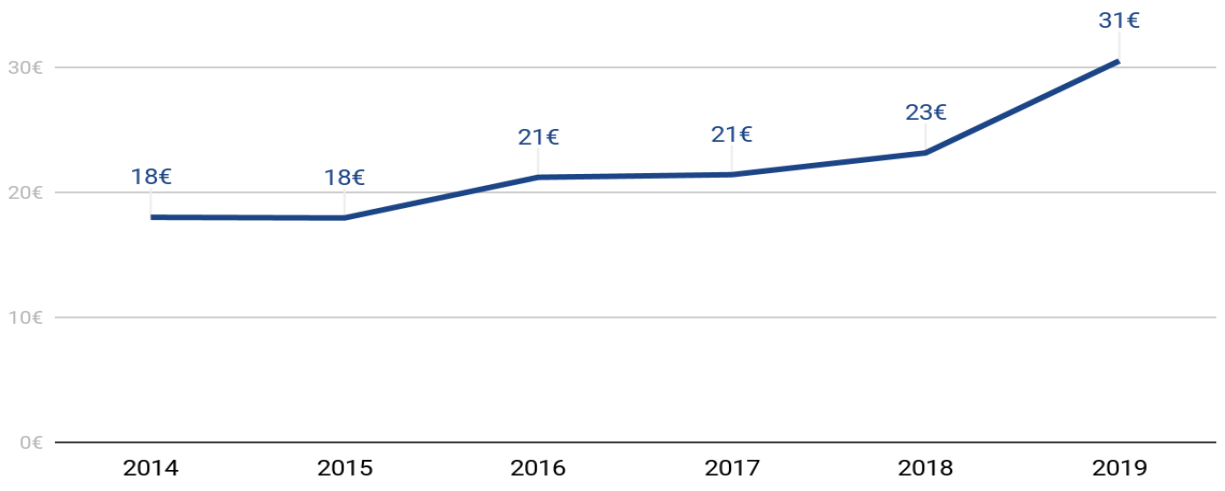

Gráfico 1/ Evolución gasto medio por visitante. Elaboración propia. 2020.

Las tareas a desarrollar dentro de la Ruta del Vino como Patronat, no solamente se contemplan las tareas vinculadas con la promoción turística, sino, tal como se ha mencionado anteriormente, con la planificación, la gestión y también el análisis de los resultados (Silveira, Silveria, 2009; Ivars-Baidal, Rebollo, 2019). El análisis de los resultados es clave para la planificación de las acciones a desarrollar en el futuro. Por ello, una de las acciones que se ha realizado es un análisis DAFO sobre la ruta, tal como se muestra en la tabla 2. 


\begin{tabular}{|c|c|}
\hline DEBILIDADES & AMENAZAS \\
\hline $\begin{array}{l}\text { - El Consejo Regulador de la DO Empordà de- } \\
\text { limita un territorio relativamente pequeño, } \\
\text { con tan solo } 2.000 \text { hectáreas de viñedo. } \\
\text { Cuenta con tan solo } 51 \text { bodegas, la mayoría } \\
\text { de ellas pequeñas empresas familiares. Estas } \\
\text { disponen de recursos limitados para dedicar } \\
\text { a la promoción y comunicación, así como al } \\
\text { enoturismo. } \\
\text { - Los vinos de la D.O. Empordà gozan de pres- } \\
\text { tigio y reconocimiento a nivel catalan, pero } \\
\text { aún son eminentemente desconocidos a ni- } \\
\text { vel internacional. } \\
\text { - Los vinos de la D.O. Empordà no se apalan- } \\
\text { can en ningún territorio de marca y, conse- } \\
\text { cuentemente, no están en el imaginario co- } \\
\text { lectivo de los consumidores de vino. } \\
\text { El débil territorio de marca de los vinos de la } \\
\text { D.O. Empordà provoca que el principal moti- } \\
\text { vo de viaje de los visitantes de las bodegas } \\
\text { sea raramente el enoturismo. } \\
\text { Debido a la consolidación de la Costa Brava } \\
\text { como destino de sol y playa, la demanda tie- } \\
\text { ne un alto grado de estacionalidad. }\end{array}$ & $\begin{array}{l}\text { - Regiones próximas con larga tradición eno- } \\
\text { turística o con un terriotrio de marca viníco- } \\
\text { la consolidado internacionalmente (Penedès, } \\
\text { Priorat, etc.) } \\
\text { Otras regiones vinícolas catalanas que ac- } \\
\text { tualmente están configurando y estructuran- } \\
\text { do su oferta enoturística (Terra Alta, Alella o } \\
\text { Pla de Bages). } \\
\text { Con la expansión del enoturismo a nivel in- } \\
\text { ternacional, aparecen nuevos destinos emer- } \\
\text { gentes con precios mucho más competitivos } \\
\text { (Georgia, Albania, Croacia, etc.). }\end{array}$ \\
\hline FORTALEZAS & OPORTUNIDADES \\
\hline $\begin{array}{l}\text { - Región con un gran legado vitivinícola histó- } \\
\text { rico, desde el comercio de vinos en las co- } \\
\text { lonias greco romanas de Empúries, hasta la } \\
\text { viticultura experta de los monjes del monas- } \\
\text { terio de St. Pere d Rodes } \\
\text { - Como destino enoturístico, las bodegas ven } \\
\text { reforzada su oferta con un amplio sector tu- } \\
\text { rístico consolidado y de calidad, desde ho- } \\
\text { teles, restauración, tiendas, equipamientos } \\
\text { culturales, empresas de actividades, etc. } \\
\text { Alto grado de sinergias entre diferentes } \\
\text { agentes enoturísticos. } \\
\text { La consolidación de la oferta enoturística ha } \\
\text { servido para fijar la población en zonas con } \\
\text { riesgo de abandono, así como también se ha } \\
\text { convertido en polo de atracción para empre- } \\
\text { sas y profesionales del sector. }\end{array}$ & $\begin{array}{l}\text { - La proximidad con la Costa Brava, zona turísti- } \\
\text { ca con gran afluencia de visitantes } \\
\text { Como consecuencia de la pandemia de la Co- } \\
\text { vid19 el turismo de hiper proximidad es ten- } \\
\text { dencia, que representa el } 64 \% \text { de los visitan- } \\
\text { tes de las bodegas. } \\
\text { El nuevo estudio de posicionamiento que está } \\
\text { realizando la D.O. Empordà a fin de consolidar } \\
\text { un apalanque de marca. } \\
\text { La transversalidad del enoturismo con otros } \\
\text { segmentos de mercado (turismo cultural, de } \\
\text { naturaleza y activo, de bienestar, gastronómi- } \\
\text { co, etc) } \\
\text { El proceso de conversión que algunas bode- } \\
\text { gas ya han acabado y muchas están imple- } \\
\text { mentando a una agricultura ecológica certifi- } \\
\text { cada. }\end{array}$ \\
\hline
\end{tabular}

Tabla 2/ Análisis DAFO Ruta del Vino D.O. Empordà. Elaboración propia. 2020 
Así pues, utilizando el método CAME (corregir, afrontar, mantener y explotar) (Hall, 2008), la Ruta del Vino D.O. Empordà, juntamente con el Consejo Regulador de la D.O. Empordà trabaja para corregir las debilidades, afrontar las amenazas, mantener las fortalezas y explorar nuevas oportunidades.

En cuanto a las debilidades, el Consejo Regulador de la D.O. Empordà está elaborando un estudio de marca que servirá para empezar a consolidar un imaginario colectivo de cómo son los vinos de esta región vinícola, hecho que ayudará a su mejor comunicación, comprensión y difusión. Asimismo, la Ruta del Vino D.O. Empordà está organizando diferentes iniciativas para la desestacionalización, como la campaña de comunicación Vívid, un festival que tiene lugar en abril; las campañas vinculadas con actividades de vendimia (a finales de agosto, septiembre y octubre) o de poda (en invierno). Igualmente, toda la comunicación generalista sobre el proyecto aproxima el enoturismo como una actividad realizable durante todo el año, con momentos interesantes y paisajes cambiantes a lo largo de las cuatro estaciones.

Por lo que refiere a las amenazas, se proponen diferentes enfoques. A nivel de turismo de proximidad y para diferenciarla de otras regiones vinícolas, se trabaja con un storytelling que busca fomentar los aspectos únicos y singulares del Empordà. A nivel internacional, conjuntamente con la Agencia Catalana de Turismo y para competir con otras destinaciones enoturísticas emergentes, se trabaja bajo el sello Enoturisme Catalunya, que pretende unificar la promoción del turismo del vino bajo una sola marca para darle más fuerza a nivel internacional.

A fin de mantener el legado vitivinícola histórico como una fortaleza de la Ruta del Vino D.O. Empordà se utiliza un storytelling donde éste se pone de manifiesto en todo tipo de soportes y formatos, desde folletos promocionales, copywriting de las diferentes webs, compra de imágenes, campañas de publicidad y branded content, etc. Además, se propicia la creación e organización de espacios donde puedan surgir sinergias entre los actores enoturísticos y los stakeholders turísticos de la Costa Brava, a fin de fortalecer la oferta.

En cuanto a explorar nuevas oportunidades, se organizan diferentes actividades para potenciar el acercamiento de los visitantes alojados en la Costa Brava y desconocedores de la riqueza enoturística en el interior. Además, a consecuencia de la pandemia global de la covid19 y al replanteamiento global del turismo a un terreno de hiperporximidad, el mercado local cobra un peso fundamental y habrá que trabajarlo en consequencia. Finalmente, se está comunicando de manera activa las bodegas que ya trabajan bajo criterios de agricultura ecológica, así como fomentando la formación en cuanto a temas de turismo sostenible. 


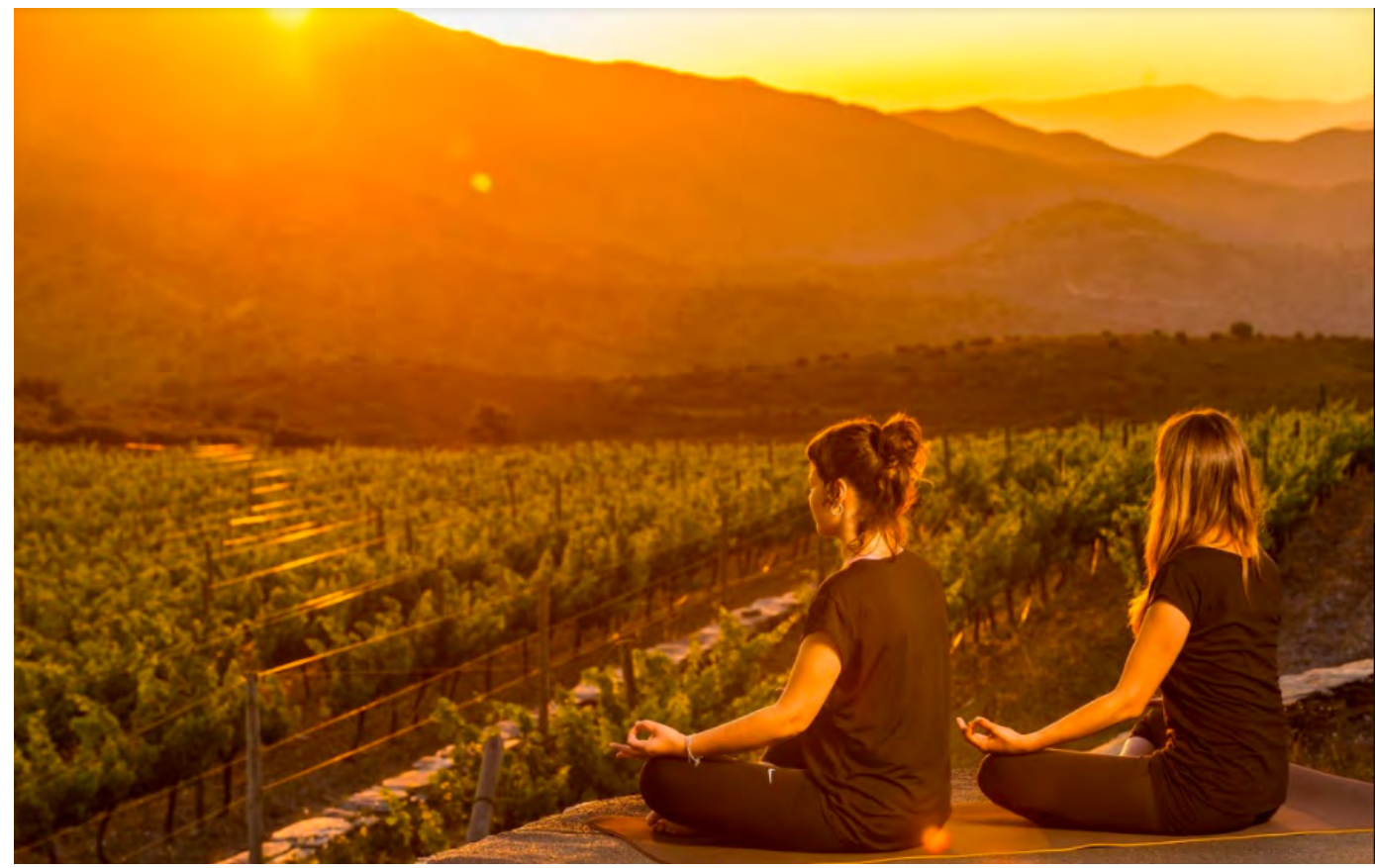

Foto 2/ loga entre viñedos. Cadaqués. Oscar Rodbag. Arxiu Imatges PTCBG. 2020

\subsection{El Plan de Acciones}

La Ruta del Vino D.O. Empordà se rige por un Plan de Acciones anual que es diseñado por el Patronato de Turismo Costa Brava Girona conjuntamente con el Comité Ejecutivo, un grupo de 13 representantes de diferentes tipologías de empresa, a fin de tener en cuenta las necesidades directas del sector y ser el máximo de operativo posible.

Este Plan de Acciones sigue los preceptos del método CAME mencionado anteriormente (Hall, 2008) y estructura sus actividades en función del público objetivo al cual van dirigidas. En algunas de estas acciones se prevé la participación directa de las empresas y entidades asociadas a la Ruta del Vino D.O. Empordà, sobretodo las de formato más comercial.

El Plan de Acciones (tabla 3) está vinculado a un presupuesto asignado y está integrado al Plan de Acciones y Objetivos anual del Patronato de Turismo Costa Brava Girona. 


\begin{tabular}{|c|c|c|}
\hline Público objetivo & Tipo de acción & Objetivo \\
\hline \multirow[t]{8}{*}{ Público final } & $\begin{array}{l}\text { Organización festival Vívid, el mes de las } \\
\text { experiencias enoturísticas (abril) }\end{array}$ & \multirow{8}{*}{$\begin{array}{l}\text { Desestacionalización, propicia- } \\
\text { ción de sinergias y creación de } \\
\text { producto enoturístico elaborado, } \\
\text { aumento del gasto por visitante, } \\
\text { difusión de la oferta enoturística, } \\
\text { inclusiva e sostenible y penetra- } \\
\text { ción de la marca. }\end{array}$} \\
\hline & $\begin{array}{l}\text { Asistencia a ferias turísticas nacionales e } \\
\text { internacionales }\end{array}$ & \\
\hline & $\begin{array}{l}\text { Edición de material promocional (mapa y } \\
\text { catálogo en catalan, castellano, inglés y fran- } \\
\text { cés) con información sobre inclusividad y } \\
\text { producción ecológica }\end{array}$ & \\
\hline & $\begin{array}{l}\text { Gestión diaria de contenido en redes socia- } \\
\text { les (Facebook, Instagram y Twitter) }\end{array}$ & \\
\hline & $\begin{array}{l}\text { Actualización de la información de todos } \\
\text { los asociados en la web institucional www. } \\
\text { costabrava.org }\end{array}$ & \\
\hline & $\begin{array}{l}\text { Creación y actualización del portal web ins- } \\
\text { piracional de experiencias enogastronómi- } \\
\text { cas www.enogastronomia.costabrava.org }\end{array}$ & \\
\hline & $\begin{array}{l}\text { Creación de merchandising promocional } \\
\text { (llaveros con corcho del Empordà), sacacor- } \\
\text { chos, dropstops, etc. }\end{array}$ & \\
\hline & $\begin{array}{l}\text { Publicidad y compra de contenidos en me- } \\
\text { dios especializados pero también de viajes, } \\
\text { ocio, cultura o gastronomía, tanto online } \\
\text { como en papel }\end{array}$ & \\
\hline \multirow[t]{5}{*}{ Prescriptores } & $\begin{array}{l}\text { Organización de viajes de prensa, tanto es- } \\
\text { pecializada como generalista turística }\end{array}$ & \multirow{5}{*}{$\begin{array}{l}\text { Creación de contenido tanto on- } \\
\text { line como en papel, difusión de } \\
\text { la oferta enoturística inclusiva y } \\
\text { sostenible tanto en medios como } \\
\text { a través de los profesionales del } \\
\text { sector, potenciando un storyte- } \\
\text { lling diferenciador, penetración de } \\
\text { la marca. }\end{array}$} \\
\hline & $\begin{array}{l}\text { Organización de viajes de bloggers, insta- } \\
\text { gramers y creadores de contenido online }\end{array}$ & \\
\hline & $\begin{array}{l}\text { Organización de jornadas para las oficinas } \\
\text { de turismo del territorio }\end{array}$ & \\
\hline & $\begin{array}{l}\text { Organización de presentaciones y catas de } \\
\text { vino, tanto presenciales como en nuevos } \\
\text { formatos virtuales, nacionales como interna- } \\
\text { cionales para periodistas }\end{array}$ & \\
\hline & $\begin{array}{l}\text { Organización de jornadas para las oficinas } \\
\text { de turismo del territorio }\end{array}$ & \\
\hline
\end{tabular}




\begin{tabular}{|c|c|c|}
\hline \multirow[t]{4}{*}{ Intermediación } & $\begin{array}{l}\text { Organización de viajes de familiarización } \\
\text { para AAVV y TTOO, tanto especializados } \\
\text { como genéricos que trabajen también eno- } \\
\text { turismo como oferta complementaria }\end{array}$ & \multirow{4}{*}{$\begin{array}{l}\text { Soporte a la comercialización de } \\
\text { las empresas y entidades asocia- } \\
\text { das a la Ruta del Vino D.O. Em- } \\
\text { pordà, identificación de las AAVV } \\
\text { y TTOO del sector enoturístico, } \\
\text { difusión de la oferta enoturística, } \\
\text { inclusiva e sostenible y penetra- } \\
\text { ción de la marca. }\end{array}$} \\
\hline & $\begin{array}{l}\text { Asistencia en workshops nacionales e inter- } \\
\text { nacionales }\end{array}$ & \\
\hline & $\begin{array}{l}\text { Organización de presentaciones y catas de } \\
\text { vino, tanto presenciales como en nuevos } \\
\text { formatos virtuales, nacionales como interna- } \\
\text { cionales para AAVV y TTOO }\end{array}$ & \\
\hline & Asistencia a ferias turísticas profesionales & \\
\hline \multirow[t]{8}{*}{ Marketing interno } & $\begin{array}{l}\text { Formación para el sector (inglés y francés } \\
\text { para el enoturismo, dinamización de RRSS, } \\
\text { maximizar }\end{array}$ & \multirow{2}{*}{$\begin{array}{l}\text { Mejora de la competitividad, inno- } \\
\text { vación en las experiencias y pro- } \\
\text { ductos enoturísticos, propiciación } \\
\text { de sinergias. }\end{array}$} \\
\hline & $\begin{array}{l}\text { Viajes de benchmark a otros destinos enotu- } \\
\text { rísticos de referencia }\end{array}$ & \\
\hline & $\begin{array}{l}\text { Organización de networking entre los asocia- } \\
\text { dos de la Ruta del Vino D.O. Empordà y los de } \\
\text { los otros clubs de marketing }\end{array}$ & $\begin{array}{l}\text { Propiciación de sinergias y crea- } \\
\text { ción de producto enoturístico } \\
\text { elaborado (como el enocaiac, los } \\
\text { maridajes con flores silvestres o } \\
\text { las visitas enoturísticas del Mo- } \\
\text { nasterio de St. Pere de Rodes). }\end{array}$ \\
\hline & $\begin{array}{l}\text { Visitas comerciales y de asesoramiento, tan- } \\
\text { to a empresas privadas como ayuntamientos } \\
\text { y demás entidades públicas }\end{array}$ & $\begin{array}{l}\text { Estructuración de la oferta eno- } \\
\text { turística, asesoramiento al sector } \\
\text { (visitas para orientar a las bode- } \\
\text { gas que están iniciando la activi- } \\
\text { dad turística) }\end{array}$ \\
\hline & $\begin{array}{l}\text { Reunión del Comitè Ejecutivo, organización } \\
\text { de grupos de trabajo temáticas y otros espa- } \\
\text { cios de debate }\end{array}$ & $\begin{array}{l}\text { Propiciación de sinergias y crea- } \\
\text { ción de producto enoturístico ela- } \\
\text { borado (trabajando codo a codo } \\
\text { con el sector en todo momento) }\end{array}$ \\
\hline & $\begin{array}{l}\text { Creación de merchandising para uso de los } \\
\text { asociados con el logo de la Ruta del Vino D.O. } \\
\text { Empordà (copas, cubiteras, escupideras, etc) }\end{array}$ & Penetración de marca. \\
\hline & $\begin{array}{l}\text { Catalogación de la oferta enoturística según } \\
\text { criterios de inclusividad y sostenibilidad }\end{array}$ & $\begin{array}{l}\text { Estructuración de la oferta eno- } \\
\text { turística, asesoramiento al sector, } \\
\text { difusión de la oferta enoturística, } \\
\text { inclusiva e sostenible y penetra- } \\
\text { ción de la marca. }\end{array}$ \\
\hline & $\begin{array}{l}\text { Recopilación y tratamiento de datos de visi- } \\
\text { tantes }\end{array}$ & $\begin{array}{l}\text { Inteligencia de mercado, previsión } \\
\text { de acciones, estudio de la deman- } \\
\text { da. }\end{array}$ \\
\hline
\end{tabular}


Este Plan de Acciones es la hoja de ruta que se sigue durante el año, acordada por todo el sector enoturístico vinculada a la ruta. Tal como sugieren los expertos en planificación (Díez Santo, 2011), se trata de un documento que tiene que ser vivo y flexible, para poderse adaptar en todo momento al cambiante mercado y a las tendencias volátiles, sobretodo este 2020 cuando ha sido imprescindible adaptar mucha de la actividad prevista a formatos híbridos o directamente virtuales, así como acercar muchas de las acciones a mercados más próximos.

Como se puede apreciar en la tabla 3, las acciones van dirigidas a cuatro grupos de acción o públicos: el público final, los prescriptores, los intermediarios y el marketing interno. El marketing interno es un elemento clave (Coria, 2015; Vergara et al., 2016). Cómo se ha podido ver, los integrantes de la Ruta del Vino D.O. Empordà son agentes de distintos sectores, tanto del sector turístico (hoteles, restaurantes, empresas de servicios), como del sector agroalimentario (bodegas) por lo que las estrategias de cohesión internas son fundamentales para garantizar la implicación de todos generando sentimientos de pertenencia e identidad.

\section{Resultados y conclusión}

El presente artículo se ha empezado a desarrollar desde la perspectiva más académica para ir paulatinamente tomando la forma de un ensayo y de un estudio de caso eminentemente empírico. Esto cobra sentido en la medida en la que existe un volumen muy limitado de textos que abordan el marketing propiamente como herramienta de gestión turística. Por lo tanto, la principal aportación es la fundamentación de este aspecto a través de la práctica profesional.

Saliendo de la malinterpretación de marketing como sinónimo de promoción, se identifican áreas de actuación que a menudo requieren de la coordinación de varios stakeholders con los cuales la DMO no puede eludir la responsabilidad de cooperar: creación de políticas, cooperación entre los empresarios del lugar pertenezcan o no directamente al sector turístico o la población local, por poner algunos ejemplos. Cuando el producto es el territorio (insistimos, humano y físico), cualquiera de ellos desempeña un papel importante en aspectos como la configuración del producto turístico y en la generación y mantenimiento de los imaginarios.

En este sentido, cabe destacar como punto importante que una ruta turística convenientemente gestionada contribuye positivamente a la calidad de vida de los residentes también en aquello inmaterial: crea cohesión territorial, sinergias a nivel económico y, en general, un sentimiento de pertenencia a la colectividad. Además, en el campo laboral, no solamente se crean oportunidades sino que existe la posibilidad de atraer talentos ajenos al territorio.

El rol de las administraciones públicas ha sido determinante en el desarrollo del enoturismo en varias destinaciones vinícolas (Serrano, 2015). Tal como se ha visto en la revisión de la literatura, el hecho de utilizar una ruta o itinerario como elemento de gestión da a la DMO una herramienta para mejorar la cohesión entre los diferentes agentes implicados. Fijándonos en el caso concreto de la Costa Brava, la Ruta del Vino D.O. Empordà, su existencia diversifica el producto, lo cual tiene dos efectos positivos destacables para los propósitos de este artículo. Primero, reparte los flujos turísticos en el tiempo (contribuye a desestacionalizar la temporada alta estival) y en el espacio (descentraliza el turismo de la costa hacia el interior). Segundo, contribuye al cambio en el perfil típico del turista vacacional de sol y playa, ofreciéndole alternativas que le permitan conocer más a fondo el territorio desde una perspectiva del 
ocio humanista. Asimismo, se logra también atraer a otro perfil de visitante que hace uso de un perfil socioeconómico medio o alto.

Vemos, por tanto, que utilizar las herramientas del marketing holístico para la gestión de un destino (ruta) de forma integral, de hecho, acaba contribuyendo al objetivo genérico generar demanda y aumentar las ventas. El proceso que hay detrás, sin embargo, es mucho más complejo que una campaña publicitaria, por lo que ésta(s) acaban conteniéndose en planes estratégicos a varios años vista que, además, deben coordinarse com las otras DMOs e instituciones operando en el mismo marco. Uno de los retos es, justamente, cómo aplicar herramientas que permitan mejorar el trabajo interno dentro de la ruta, convirtiendo el marketing interno en un aspecto clave.

Futuras investigaciones deberían contemplar la aplicación de otros métodos de estudio que permitan ir más allá en el estudio de caso, cómo las entrevistas con los agentes o bien la observación in situ de las dinámicas que se generan en el territorio.

\section{Referencias bibliográficas}

- Afshardoost, M., Eshaghi, M.S. (2020). Destination image and tourist behavioural intentions: a meta-analysis. Tourism Management, 81. https://doi.org/10.1016/j.tourman.2020.104154

- $\quad$ Amilhat-Szary, A-L., Guyot, S. (2009). Turismo transfronterizo en los Andes Centrales: prolegómenos sobre una geopolítica del turismo. Si Somos Americanos, Revista de Estudios Transfronterizos, IX(2), 63-100. http://www.redalyc.org/articulo.oa?id=337930336005

- Aulet, S. (2010). Itinerarios culturales en Cataluña: de la idea al proyecto real. In Itinerarios culturales: la experiencia del camino de los diamantes= Itinerarios culturais: a experiencia do caminho dos diamantes, 55-69. Girona: Documenta Universitaria.

- $\quad$ Aulet, S., Mundet, L., \& Vidal, D. (2017). Monasteries and tourism: interpreting sacred landscape through gastronomy. Revista Brasileira de Pesquisa em Turismo, 11(1), 175-196.

- $\quad$ Aulet, S., Vidal-Casellas, D., \& Majó, J. (2019). Community development through gastronomic tourism. The Routledge handbook of gastronomic tourism, 242-251. Oxford: Routledge.

- Barron, L. (2017, August 30). "Unesco-cide": does world heritage status do cities more harm than good? The Guardian, online. Recuperado de https://www.theguardian.com/cities/2017/aug/30/ unescocide-world-heritage-status-hurt-help-tourism

- Barroso Castro, C., Martín Armario, E., Martín Ruiz, D. (2007). The influence of market heterogeneity on the relationship between a destination's image and tourists' future behaviour. Tourism Management, 28(1), 175-187. https://doi.org/10.1016/j.tourman.2005.11.013

- Bramwell, B., Rawding, L. (1994). Tourism marketing organizations in industrial cities. Tourism Management, 15(6), 425-434. scihubtw.tw/10.1016/0261-5177(94)90063-9

- Calderón Puerta, D. M., Arcila Garrido, M., \& López Sánchez, J. A. (2018). La rutas e itinerarios turístico-culturales en los portales oficiales de turismo de las Comunidades Autónomas españolas. Revista de Estudios Andaluces, 35, 123-145.

- Carrillo Sánchez, Laura Grisel; García Calderón, Carola Isabel (2019). Gestión de la marca Ciudad de México (CDMX) y su impacto económico. Questiones Publicitarias, 24, pp. 77-85 https://doi. org/10.5565/rev/ap.320

- $\quad$ Chen, C. F., \& Chen, P. C. (2010). Resident attitudes toward heritage tourism development. Tourism Geographies, 12(4), 525-545. 
- Chias, J. (2004). El Negocio de la felicidad : desarrollo y marketing turístico de países, regiones, ciudades y lugares. Madrid : Prentice Hall.

- Consejo de Europa (2015). Cultural routes management: from theory to practice. Estrasburgo: Council of Europe Publishing.

- Coria, C. (2015). La importancia del marketing interno en las empresas de servicio. Universidad de Buenos Aires. Tesis doctoral.

- Díez Santo, D. (2011). La planificación estratégica en espacios turísticos de interior: Claves para el diseño y formulación de estrategias competitivas. Investigaciones Turísticas, 1, enero-junio, p. 69-92.

- du Cros, H. (2008). Too much of a good thing? Visitor congestion management issues for popular world heritage tourist attractions. Journal of Heritage Tourism, 2(3), 225-238. https://doi. org/10.2167/jht062.0

- Ellis, N., Fitchett, J., Higgins, M., Jack, G., Lim, M., Saren, M., Tadajewski, M. (2010). Marketing. A critical textbook. London: SAGE Publications

- European Travel Commission, Organización Mundial del Turismo (2017). Handbook on marketing transnational tourism themes and routes. Madrid: Organización Mundial del Turismo.

- González Vázquez, D. (2018). Les rutes de l'exili del MUME. Una dècada connectant espais de memòria a la Catalunya transfronterera. Ebre, (8), 279-308. https://dialnet.unirioja.es/servlet/articulo? codigo $=6448549$

- Gross, T. (2011). 51 Toomas Gross: Divided over Tourism: Zapotec Responses to Mexico's "Magical Villages Program" Divided over Tourism: Zapotec Responses to Mexico's 'Magical Villages Program'. Anthropological Notebooks, 17(3), 51-71. Recuperado de http://www.drustvo-antropologov. si/AN/PDF/2011_3/Anthropological_Notebooks XVII 3 Gross.pdf

- Hall, C. M. (2008). Tourism planning: Policies, processes and relationships. London: Pearson Education.

- Hernández, M. G. (2000). Turismo y medio ambiente en ciudades históricas. De la capacidad de acogida turística a la gestión de los flujos de visitantes. Anales de geografía de la Universidad Complutense, Vol. 2000 (20), p. 131-148.

- IDESCAT (2020). Institut d'estadística de Catalunya. www.idescat.cat.

- Ivars-Baidal, J., \& Rebollo, J. F. V. (2019). Planificación turística en España. De los paradigmas tradicionales a los nuevos enfoques: planificación turística inteligente. BAGE: Boletín de la Asociación de Geógrafos Españoles, (82), 7.

- Kotler, P., \& Armstrong, G. (2008). Principios de marketing. Madrid : Esic editorial.

- Leal Londoño, M. D. P. (2013). Turismo gastronómico y desarrollo local en Cataluña: el abastecimiento y comercialización de los productos alimenticios. Universidad de Barcelona: Tesis doctoral.

- Llorca Hernández, F.J. (2018). Gobernanza turística de itinerarios culturales. En Martín-Cabello, A., García-Manso, A., Anta Félez, J.L. (coord.), I Congreso Internacional de Estudios Culturales Interdisciplinares. Cultura e identidad en un mundo cambiante (p. 387-396). OMM Press

- Majdoub, W. (2010). Analyzing cultural routes from a multidimensional perspective. Almatourism-Journal of Tourism, Culture and Territorial Development, 1(2), 29-37.

- Mitchell, R., Hall M. (2006). Wine Tourism Research: The State of Play.

- Tourism Review International, 9(4), 307-332Morillo Palomo, C. (2015). Creación y posocionamiento de la Barcelona postolímpica como marca ciudad (Tesis doctoral, Universitat Ramon Llull). https:/l tdx.cat/handle/10803/352472\#page $=191$

- Nogué i Font, J. (1992). Turismo, percepción del paisaje y planificación del territorio. Estudios Turísticos, 1992, núm. 115, p. 45-54. 
- $\quad$ OMT (2019). Guía para el desarrollo del turismo gastronómico. Madrid: OMT. https://www.e-unwto. org/doi/pdf/10.18111/9789284420995

- Patronat de Turisme Costa Brava Girona (2011). Pla estratègic turístic de les Comarques Gironines (2011 - 2015). Girona: Patronat de Turisme Costa Brava Girona.

- Sánchez, I. R. (2015). Identidad, patrimonio y turismo; interrelación en la valorización de autenticidad del producto turístico. Vía innova, (2), 9-13.

- Schmitz, M., Espinosa, A. (2015). Impacto de la información disonante y consistente sobre la campaña Marca Perú en la identidad nacional y en bienestar social. Universitas Psychologica, 14(1), 329-338. http://dx.doi.org/10.11144/Javeriana.upsy13-5.iidc

- Serrano, D. (2015). Enoturisme com a fenomen de desenvolupament local al món i a Catalunya

- Sheth, J.N., Sisodia, R.S. (eds.) (2015). Does marketing need a reform? Fresh perspectives on the future. Londres: Routledge.

- Silveira, J. M., \& Silveira, C. E. (2009). La evolución del marketing de destinos. Su sinergia con la planificación turística. Estudios y perspectivas en turismo, 18(5), 530-545.

- $\quad$ Sparks, B., \& Malady, J. (2006). Emerging wine tourism regions: Lessons for development. Global wine tourism: Research, management and marketing, 67-79.

- Timothy, D. J. (Ed.). (2015). Heritage cuisines: traditions, identities and tourism. Oxford: Routledge.

- Tolosa, LI. (2017). Marketing del enoturismo. 12 errores habituales. 12 propuestas alternativas

- Toselli, C. (2006). Algunas reflexiones sobre el turismo cultural. Pasos revista de turismo y patrimonio cultural, 4(2), 175-182.

- Vergara, M. C., Marín, A. Á., Oteiza, K. M., Alvarado, N., \& Codoceo, M. J. (2016). Impacto del marketing interno en el desarrollo de innovación: la co-creación en el sector turístico de La Serena-Chile. Estudios y perspectivas en turismo, 25(2), 203-222 


\section{CURRICULUM VITAE}

Neus Crous-Costa, Mtra.

Máster en Planificación y Dirección del Turismo por la Universidad de Girona. Investigadora en formación en materia de turismo y desarrollo personal. Otras líneas de investigación y docencia incluyen turismo como herramienta para el diálogo, gestión e interpretación del patrimonio, turismo espiritual y cooperación. Anteriormente ha trabajado en los campos de la gestión turística en museos y en consultoría, entre otros. Colabora en redes internacionales como la Organización de Ciudades Patrimonio de la Humanidad - Secretariado Regional de Asia-Pacífico y Stay Grounded.

\section{Marta Casas i Serrabassa, Mtra.}

Diplomada en Turismo por la Universidad de Girona, Postgrado en Wine \& Business Marketing por la Escuela Superior de Hostelería de Barcelona y WSET nivel 2. Actualmente técnica de marketing en el Patronato de Turismo Costa Brava Girona como product manager del club de marketing de la Ruta del Vino D.O. Empordà. 